\title{
On the interpretation of energy and energy fluxes of nonlinear internal waves: an example from Massachusetts Bay
}

\author{
By A. SCOTTI $I^{1}, R$. BEARDSLEY AND B. BUTMAN ${ }^{3}$ \\ ${ }^{1}$ Department of Marine Sciences, UNC, Chapel Hill, NC 27599-3300, USA \\ ${ }^{2}$ Department of Physical Oceanography, WHOI, Woods Hole, MA 02543, USA \\ ${ }^{3}$ US Geological Survey, Woods Hole, MA 02543, USA
}

(Received 27 March 2006 and in revised form 22 May 2006)

A self-consistent formalism to estimate baroclinic energy densities and fluxes resulting from the propagation of internal waves of arbitrary amplitude is derived using the concept of available potential energy. The method can be applied to numerical, laboratory or field data. The total energy flux is shown to be the sum of the linear energy flux $\int u^{\prime} p^{\prime} \mathrm{d} z$ (primes denote baroclinic quantities), plus contributions from the non-hydrostatic pressure anomaly and the self-advection of kinetic and available potential energy. Using highly resolved observations in Massachusetts Bay, it is shown that due to the presence of nonlinear internal waves periodically propagating in the area, $\int u^{\prime} p^{\prime} \mathrm{d} z$ accounts for only half of the total flux. The same data show that equipartition of available potential and kinetic energy can be violated, especially when the nonlinear waves begin to interact with the bottom.

\section{Introduction}

Observations of nonlinear internal waves (NLIWs) in the ocean are rapidly growing in number (Apel, Ostrovsky \& Stepanyants 1995). Remote sensing techniques show that NLIWs of depression are a common feature over most continental shelves (Jackson 2004), but there are also many examples of NLIWs propagating across entire basins, such as the South China Sea (Ramp et al. 2004; Duda et al. 2004) or the Andaman Sea (Osborne \& Burch 1980). In addition, recent observations indicate that near-bottom waves of elevation might be just as widespread (Klymak \& Moum 2003; Hosegood \& van Haren 2004; Scotti \& Pineda 2004). NLIWs have important effects on many problems, ranging from the biology of pelagic organisms (Pineda 1991) to sound propagation and attenuation (Apel et al. 1997), and have become a subject of numerous field and theoretical studies, which are summarized well in Helfrich \& Melville (2006).

An interesting property of NLIWs is that they can very efficiently package and transport energy across considerable distances. Once they reach shallow waters, the energy can then be applied to mixing or to drive along-shore currents via a radiation stress divergence mechanism. It is therefore important to quantify the baroclinic energy flux associated with NLIWs. While baroclinic fluxes are generally small when compared to barotropic fluxes, they nontheless represent energy that is 'ready' for mixing, whereas away from boundaries barotropic energy has first to go through a cascade process in order to cause mixing (see e.g. Laurent \& Garrett 2002). 
Jeans \& Sherwin (2001) were among the first to attempt to quantify the energy fluxes associated with NLIWs, including the nonlinear contributions (i.e. contributions from terms other than $\int u^{\prime} p^{\prime} \mathrm{d} z$, where primes denote baroclinic quantities). They considered NLIWs propagating along the Portuguese shelf using a combination of shipboard acoustic Dopple current profile (ADCP) measurements for currents and conductivitytemperature-depth (CTD) casts and fixed thermistor chains for density. Their analysis suffered from two shortcomings: one related to the accuracy of ADCP-measured currents in the presence of short-wavelength waves discussed in Scotti et al. (2005), and the other due to the lack of a unifying framework to estimate fluxes, which resulted in potentially confusing mesoscale-mediated changes in stratification for NLIWs-caused mixing. More recently, Lien et al. (2005) and Chang et al. (2006) looked at the energetics and energy fluxes for NLIWs in the South China Sea. They report a much larger value for the energy than predicted by the Garret-Munk spectrum and an almost complete dissipation of the waves as they leave the deep part of the basin. However, they used linear-wave theory to account for deficiencies in the vertical coverage of the water column, and they apparently did not take into account the already mentioned problem with ADCPs. Moreover, they did not include nonlinear fluxes (to be defined below), which is particularly significant, as they represent a sizable component of the total flux.

These examples highlight the theoretical and observational challenging nature of nonlinear internal waves. In this paper we develop a consistent framework to diagnose the energetics of internal waves. It recovers the standard treatment of Nash, Alford $\&$ Kunze (2005) in the limit of small waves, but it applies to waves of any amplitude and wavelength. To examine the importance of each term making up the total flux, we apply the framework to NLIWs observed in Massachusetts Bay, where a high-quality comprehensive dataset exists which allows us to calculate all of the terms involved in the energy equation.

\section{Energy equation for a stratified fluid}

Our treatment of the energy problem for NLIWs represents a synthesis of the concept of available potential energy (APE) as discussed in Shepherd (1993) and Winters et al. (1995). The idea of APE goes back to the early works of Margules (1903) and Lorenz (1955), who showed that in order to be meaningful, potential energy has to be referenced to an appropriately defined background potential energy.

Let $\bar{\rho}=\bar{\rho}(z, t)$ be a properly defined density reference state, strictly decreasing in $z$ (stable stratification) and possibly time dependent, and let $z^{*}(s, t)$ be such that $\bar{\rho}\left(z^{*}(s, t), t\right)=s$. Let $\min \rho<\rho_{0}<\max \rho$ be a given density reference and define

$$
\psi=\int_{\rho_{0}}^{\rho} g z^{*} \mathrm{~d} s,
$$

where $\rho=\rho(\boldsymbol{x}, t)$ is the density of the fluid. The spatial dependence of $\psi$ depends only on the spatial dependence of the density field whence it follows that $\nabla \psi=g z^{*} \nabla \rho$. Let the evolution of the fluid be governed by the Navier-Stokes equations for a fluid of variable density in the Boussinesq approximation. It is a matter of simple algebra to show that from conservation of mass $\psi$ obeys

$$
\frac{\mathrm{D} \psi}{\mathrm{D} t}=\kappa \nabla^{2} \psi-\kappa g|\nabla \rho|^{2} \frac{\partial z^{*}}{\partial \rho}+g \int_{\rho_{0}}^{\rho} \frac{\partial z^{*}}{\partial t} \mathrm{~d} s,
$$

where $\mathrm{D} / \mathrm{D} t$ is the Lagrangian derivative and $\kappa$ the molecular diffusivity of the stratifying agent. The first term on the right-hand side is diffusion of $\psi$, the second, 
which is positive, represents the effect of irreversible mixing (see below), while the last reflects changes to the background state. Similarly, we can write

$$
\frac{\mathrm{D}}{\mathrm{D} t}(\rho g z)=\rho g w+\kappa \nabla^{2}(\rho g z)-2 g \kappa \frac{\partial \rho}{\partial z} .
$$

Here the terms on the right are the buoyancy flux, diffusion of total potential energy and work done against gravity by diffusive processes. If we subtract (2.2) from (2.3) and add the equation for kinetic energy, we arrive at the following equation:

$$
\begin{aligned}
\frac{\partial}{\partial t}\left(E_{k}+\rho g z-\psi\right)=\nabla^{2}\left(\nu E_{k}+\right. & \kappa(\rho g z-\psi))-\boldsymbol{u} \cdot \nabla(J)-\epsilon_{k} \\
& +\kappa g|\nabla \rho|^{2} \frac{\partial z^{*}}{\partial \rho}-2 g \kappa \frac{\partial \rho}{\partial z}-g \int_{\rho_{0}}^{\rho} \frac{\partial z^{*}}{\partial t} \mathrm{~d} s,
\end{aligned}
$$

where $E_{k}$ is the kinetic energy density, $\epsilon_{k}$ the kinetic energy dissipation term, $v$ the molecular viscosity and $J=p+E_{k}+\rho g z-\psi$. Finally, since

$$
\psi=\int_{\bar{\rho}}^{\rho} g z^{*} \mathrm{~d} s+\bar{\rho} g z-\rho_{0} g z_{0}(t)-g \int_{z_{0}(t)}^{z} \bar{\rho} \mathrm{d} z^{\prime},
$$

with $z_{0}(t)=z^{*}\left(\rho_{0}, t\right),(2.4)$ can be recast into the final form of the energy equation

$$
\begin{aligned}
\frac{\partial}{\partial t}\left(E_{k}+E_{p}\right)+\nabla\left(\boldsymbol{u}\left(P+E_{k}+E_{p}\right)\right) & =\nabla^{2}\left(v E_{k}+\kappa E_{p}\right) \\
-\epsilon_{k} & -\epsilon_{p}-2 g \kappa \frac{\partial}{\partial z}(\rho-\bar{\rho})-g \int_{\bar{\rho}}^{\rho} \frac{\partial z^{*}}{\partial t} \mathrm{~d} s,
\end{aligned}
$$

where

$$
\left.\begin{array}{l}
P=p+g \rho_{0} z_{0}(t)+\int_{z_{0}(t)}^{z} g \bar{\rho} \mathrm{d} z^{\prime}, E_{p}=g \int_{\bar{\rho}}^{\rho}\left(z-z^{*}\right) \mathrm{d} s, \\
\epsilon_{p}=-g \kappa\left(\left.|\nabla \rho|^{2} \frac{\partial z^{*}}{\partial \rho}\right|_{\rho}-\left.|\nabla \bar{\rho}|^{2} \frac{\partial z^{*}}{\partial \rho}\right|_{\bar{\rho}}\right) .
\end{array}\right\}
$$

$P$ is the deviation from the hydrostatic pressure distribution associated with the reference state. The terms on the right of (2.6) represent diffusion, conversion of energy to internal energy (heat) due to viscous friction, energy used to irreversibly raise the centre of mass of the system by molecular mixing (a geometric interpretation of this term was given by Winters \& D'Asaro 1996) and changes in $E_{p}$ due to changes in the backgroud state. Note that (2.6) does not depend on the choice of the reference density $\rho_{0}$ and that each term is identically zero if $\boldsymbol{u}=0$ and $\rho=\bar{\rho}$.

\subsection{Properties of the energy equation}

\subsubsection{Closed systems}

For a closed system, if one chooses $\bar{\rho}$ as the adiabatic restratification of $\rho,(2.6)$ is the local version of the volume-averaged equations derived by Winters et al., and $E_{p}$ represents the available potential energy as it is commonly understood. Our derivation highlights the fact that it is possible to attach a local meaning to $E_{p}$, as already noted by Shepherd. In fact, as defined above $E_{p}(\rho, \bar{\rho}) \geqslant 0$, as long as $\bar{\rho}$ is chosen to be monotonic. Moreover, $E_{p}(\rho, \bar{\rho})=0$ if and only if $\rho=\bar{\rho}$. For small deviations from the reference state

$$
E_{p}(\bar{\rho}+\delta, \bar{\rho})=\rho_{0} \frac{g^{2}}{2 N^{2}}\left(\frac{\delta}{\rho_{0}}\right)^{2}+O\left(\delta^{3}\right),
$$


where $N^{2}=-g \partial \bar{\rho} / \partial z \rho_{0}^{-1}$ is the Brünt-Väisälä frequency of the reference state. The above relationship is exact if the reference state is linear (i.e. constant $N^{2}$ ). The mixing terms in (2.6) are globally negative, as more easily seen if one forms an equation for the background potential energy (BPE) defined as $g \rho z-E_{p}$. However, locally they can be of either sign, unless the backgroud stratification is linear. To see that, consider again a small perturbation of the reference state and expand the mixing terms. To the lowest order the expansion is equal to

$$
-\epsilon_{p}-2 g \kappa \frac{\partial}{\partial z}(\rho-\bar{\rho}) \simeq g \kappa(\rho-\bar{\rho})|\nabla \bar{\rho}|^{2} \frac{\partial^{2} z^{*}}{\partial \rho^{2}},
$$

unless the reference state is linearly stratified, in which case

$$
-\epsilon_{p}-2 g \kappa \frac{\partial}{\partial z}(\rho-\bar{\rho})=-\kappa \frac{\rho_{0} g^{2}}{2 N^{2}}|\nabla(\rho-\bar{\rho})|^{2} .
$$

In the former case, diffusion may locally increase the APE of the parcel of fluid.

\subsection{Open or semi-open systems}

In deriving (2.6), we did not make use of the assumption that $\bar{\rho}$ is connected by an adiabatic restratification to $\rho$. All we require from $\bar{\rho}$ is that it is monotonic and $\min \bar{\rho} \leqslant \min \rho<\max \rho \leqslant \max \bar{\rho}$. Thus, (2.6) describes the evolution of a family of adiabatic global invariants with a clear link to the mixing properties of the flow. As such, they are very useful as a diagnostic tool. With NLIWs in mind, the problem usually reduces to determining how much energy is available for mixing within a semi-enclosed area (e.g. shoreward of a give isobath) or calculating how much mixing occurs within a region traversed by NLIWs. In either case, determining the exact 'background' $\bar{\rho}$ may be difficult or impossible. However, as long as conditions within the domain allow the existence of an equilibrium state $(\boldsymbol{u}=0, \rho=\bar{\rho}(z))$, one can guess a reasonable value for $\bar{\rho}$, and calculate the resulting fluxes. Equation (2.6) shows that there are no 'hidden' terms resulting from the mismatch between the estimated $\bar{\rho}$ and the real one. In fact, there is more to that. Saying that a certain region of the ocean receives a given amount of baroclinic energy flux does not tell anything about the mixing occurring within it, unless something is known about the nature of the stratification within the area, reflected in the widely used formula

$$
\kappa_{t} \sim \frac{\epsilon_{k}}{N^{2}}
$$

that links kinetic energy dissipation to eddy diffusivity (Fernando 1991). The disadvantage is that calculating the flux of pseudo-energy alone only allows the evalutation of the total amount of energy dissipated by the combined mixing and turbulent kinetic energy dissipation. However, it is always possible, either by means of direct measurements, or by using a parameterization of $\epsilon_{p}$ in terms of $\epsilon_{k}$, to separate the contributions. The alternative is to use the standard energy equation

$$
\frac{\partial}{\partial t}\left(E_{k}+\rho g z\right)+\nabla\left(\boldsymbol{u}\left(p+E_{k}+\rho g z\right)\right)=\nabla^{2}\left(v E_{k}+\kappa \rho g z\right)-\epsilon_{k},
$$

which was the route followed by Chang et al. (2006). This is appropriate for linear internal waves, once the barotropic component is removed (Kunze et al. 2002; Nash et al. 2005), because the kinetic energy and APE density associated with the waves is small relative to the baroclinic internal energy $p^{\prime}$. Thus the baroclinic flux reduces to $\int u^{\prime} p^{\prime} \mathrm{d} z$. For NLIWs, as we will show below, the baroclinic energy density becomes comparable to the baroclinic internal energy (pressure) $p^{\prime}$, and thus the term 


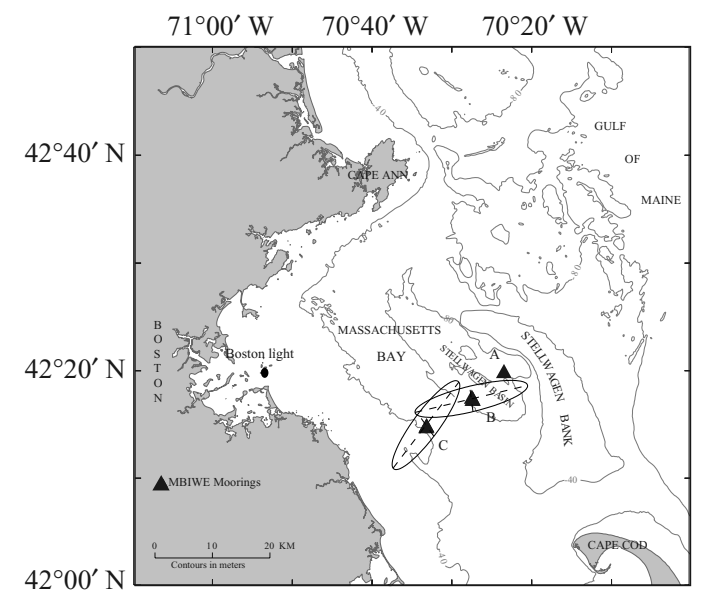

Figure 1. Map of the Massachusetts Bay/Stellwagen Bank area. The solid triangles marked $\mathrm{A}, \mathrm{B}$, and $\mathrm{C}$ show the positions of the moorings deployed during MBIWE98. The plot also shows the barotropic M2 tidal ellipses at stations B and C.

$\int \boldsymbol{u}\left(E_{k}+\rho g z\right) \mathrm{d} z$ cannot be simply dropped out. On the other hand, by not distiguishing APE from BPE, a naive calculation of the effective baroclinic flux including $u\left(E_{k}+\rho g z\right)$ amounts to calculating a small quantity by subtracting large quantities (since BPE is large), thus introducing large errors. $\dagger$ In either case, the error in the estimate is expected to be large. In addition, mixing cannot be evaluated directly, even if direct measurements of $\epsilon_{k}$ are available, but has to be parameterized. How to modify the formalism to include open systems that do not have a simple background state is a question first raised by Lorenz (1955). While the energy equation derived above holds, it is not clear how to interpret $E_{P}$. Shepherd (1993) shows that the Hamiltonian formalism can be used to consider this case, but exact expressions cannot be easily obtained, and at this moment the problem remains open.

\section{Application to Massachusetts Bay}

In 1998, a Massachusetts Bay Internal Wave Experiment (MBIWE98) was conducted to study the impact of NLIWs on sediment transport within Stellwagen Basin (figure 1). The experiment included both shipboard and moored array measurements over a period of four weeks from August 4 to September 1, 1998. More than thirty sets of NLIW packets were observed during the deployment, resulting in the most comprehensive set of observations of NLIWs in Massachusetts Bay to date (Butman et al. 2006). Moorings at B ( $85 \mathrm{~m}$ depth) and C (50 m depth) (figure 1) recorded velocity, temperature and salinity over the deployment period. ADCP measurements were postprocessed following Scotti et al. (2005) and validated with in-situ current meters. Hydrographic cruises completed during the survey period provide data to calculate the background stratification. Both at $\mathrm{B}$ and $\mathrm{C}$ the wave cycle typically can be divided into three phases. The first phase (I), which lasts approximately $40 \mathrm{~min}$, consists of a packet of large high-frequency oscillations, ranging from a few to more than 10 , followed by more random oscillations lasting for about 2 hours

$\dagger$ This problem does not exist if (2.6) is used, since the terms in the flux involving density depends explicitly on $\rho-\bar{\rho}$ and $\mathrm{BPE}$ is removed. 


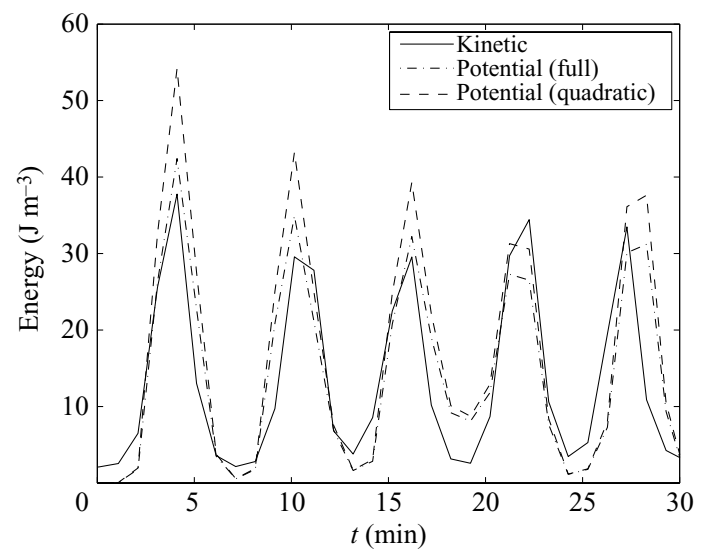

FIGURE 2. Time series of vertically averaged APE and kinetic energy $\rho\left(u^{2}+v^{2}+w^{2}\right) / 2$ of a packet of NLIWs at B. Both the full expression for $E_{p}$ and its quadratic approximation are shown.

(second phase), leaving the pycnocline displaced downward. The leading NLIWs were characterized by a mean amplitude of $15 \pm 5 \mathrm{~m}$, approximately equal to the depth of the pycnocline, with wavelengths of about $240 \mathrm{~m}$. The pycnocline slowly rebounds to its equilibrium position during the last phase. The time interval between successive sets of packets $(12.4 \mathrm{~h})$ strongly suggests that the observed NLIWs originate from the interaction of the barotropic tide with nearby Stellwagen Bank (Chereskin 1983; Trask \& Briscoe 1983). Due to instrument failure, only 25 wave cycles were observed at $\mathrm{C}$, while at $\mathrm{B}$ data were obtained over 31 cycles. The main difference between the two sites is that at $\mathrm{C}$ the waves begin to interact with the shoaling bottom.

Fluxes and energy densitites are averaged over the duration of the first phase. Thus, we aggregate the effects of several waves into a single number. Because of the variability of the NLIW field, the MBIWE dataset provides an excellent opportunity to test the relative importance of the different flux terms in the energy equation under a spectrum of nonlinear waves. The only exception to this rule is when we discuss the relative importance of high- and low-bandpassed fluxes.

\subsection{Energy density and energy fluxes}

The background stratification $\bar{\rho}$ was determined by combining data from the hydrographic cruises with average values of density recorded by the moored instruments during the periods immediately preceding the arrival of the waves. The results were found to be robust with respect to day-to-day changes in $\bar{\rho}$. Figure 2 shows a typical time series of depth-averaged available and kinetic energy for a series of NLIWs at B. For comparison, we have also plotted the leading-order term of the APE (equation (2.8)). While not large, the difference between the approximated APE and the real energy illustrates the nonlinear nature of the waves. The total energy in this particular packet is evenly distributed between kinetic and available potential energy. This appears to be a statistically robust property of the NLIWs observed at B (figure 3), despite deviations at the individual wave level and at the packet level. At C, there appears to be a shift towards more energy stored in the available potential field, probably due to a departure from weak nonlinearity caused by the shoaling bottom. The increase in overall energy densitites at $\mathrm{C}$ is similarly due to the shallowing of the water column. While equipartition is to be expected for a weakly nonlinear wave it is not clear if this property survives for strongly nonlinear waves and/or waves 


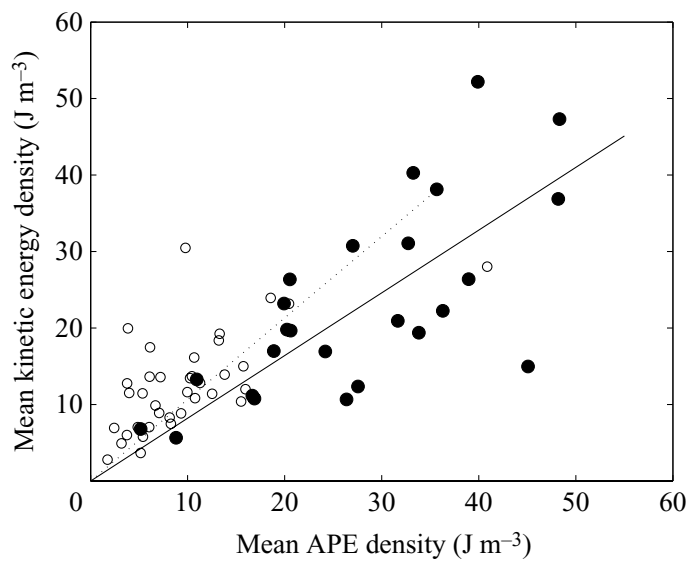

FiguRE 3. Scatter plot of APE vs. kinetic energy, depth and time averaged, of the NLIWIs observed at B (open circles) and C (solid circles). The slope of the linear regression of the data at $\mathrm{C}$ is $0.82 \pm 0.064\left(r^{2}=0.87\right)$, and at $\mathrm{B}$ is $1.06 \pm 0.088\left(r^{2}=0.8\right)$.

interacting with the bottom; the waves described in Scotti \& Pineda (2004) (analysed with the same technique) have a potential to kinetic energy ratio much larger than one.

While our approach can be used to study the depth dependence of fluxes, for ease of interpretation we consider only depth-integrated quantities, for which a partition between barotropic and baroclinic fluxes is straightfoward. To analyse the fluxes we first write the pressure $P$ as

$$
P=P_{0}(x, y, t)-g \int_{0}^{z}(\rho-\bar{\rho}) \mathrm{d} z-\rho_{0} \int_{0}^{z} \frac{\mathrm{D} w}{\mathrm{D} t} \mathrm{~d} z \equiv P_{0}+p_{h}^{\prime}+p_{n h}^{\prime},
$$

where a prime denotes baroclinic quantities (and again we have assumed a Boussinesq fluid). The first term is the pressure at the reference level $z=0$, which includes the surface tide (we have assumed for convenience that $z_{0}(t)=0$ ). To separate the baroclinic from the barotropic component of the energy it is necessary to depthintegrate (2.6) and remove the equation for the barotropic component of the energy. Since the baroclinic velocity integrates to zero and $\int\left(u-u^{\prime}\right) P_{0} \mathrm{~d} z$ is removed when we subtract the barotropic energy, we do not need to worry about this term (Nash et al. 2005). The other two terms are the baroclinic hydrostatic pressure anomaly and the non-hydrostatic pressure component due to the vertical acceleration of the fluid elements (Moum \& Smyth 2006). Thus the vertically integrated divergence term in (2.6) reduces to

$$
\int \boldsymbol{u}^{\prime} p_{\mathrm{h}}^{\prime} \mathrm{d} z+\int \boldsymbol{u} p_{\mathrm{nh}}^{\prime} \mathrm{d} z+\int \boldsymbol{u}\left(E_{k}^{\prime}+E_{p}^{\prime}\right) \mathrm{d} z \equiv F_{\mathrm{h}}+F_{\mathrm{nh}}+F_{\mathrm{nl}},
$$

where the subscripts $\mathrm{h}$, nh and $\mathrm{nl}$ mean hydrostatic, non-hydrostatic and nonlinear. $\dagger$ Note that in the nh and $\mathrm{nl}$ terms the full velocity has to be used. For the present dataset, Phase I in practice coincided with slack water, so that $\boldsymbol{u} \simeq \boldsymbol{u}^{\prime}$, but in general the advection of nonlinear energy cannot be neglected a priori.

$\dagger$ The mixed terms $\boldsymbol{u}^{\prime}\left|\boldsymbol{u}-\boldsymbol{u}^{\prime}\right|^{2} / 2$ and $\boldsymbol{u}^{\prime}\left[\left(\boldsymbol{u}-\boldsymbol{u}^{\prime}\right) \cdot \boldsymbol{u}^{\prime}\right]$ arising from the expansion of $\boldsymbol{u} E_{k}$ do not appear in (3.2) because the former integrates to zero, while the integral of the latter contributes to the barotropic energy flux. 


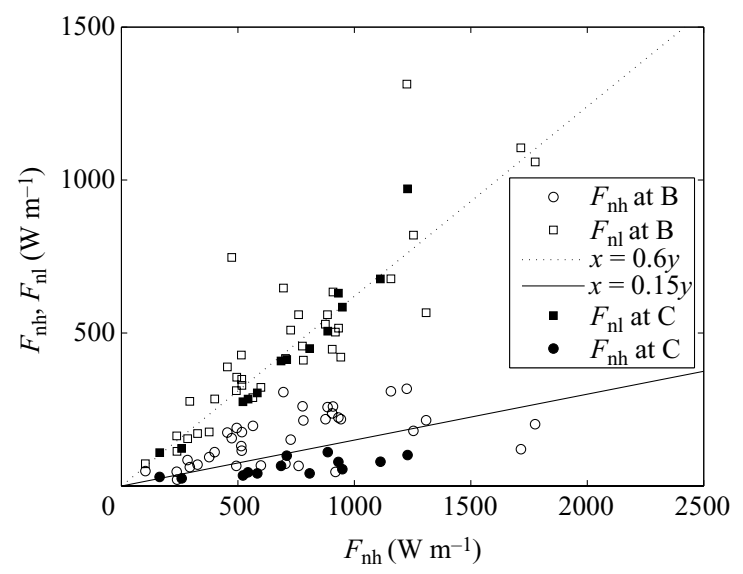

FIGURE 4. Scatter plot of hydrostatic ( $x$-axis) vs. nonlinear and nonhydrostatic ( $y$-axis) fluxes at $\mathrm{B}$ and $\mathrm{C}$. The fluxes are both depth-integrated and averaged over the duration of Phase I of the tidal cycle.

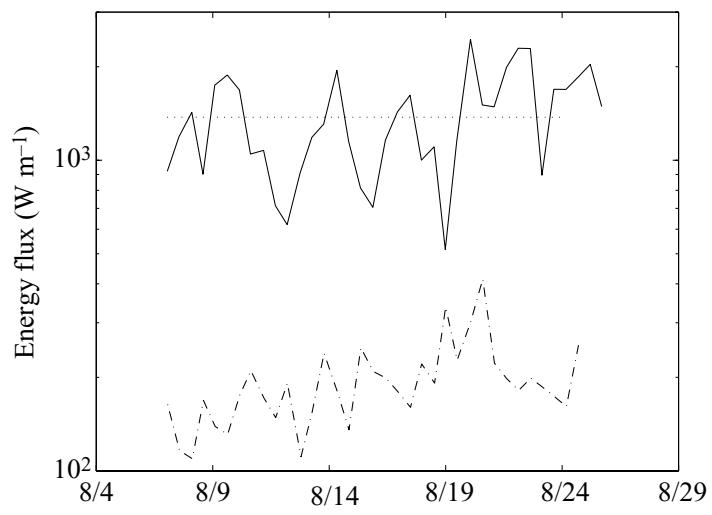

FIGURE 5. High (solid) and low-frequency (dash-dotted) component of the vertically integrated baroclinic energy fluxes at B. The high-frequency fluxes are averaged over 2 hours, while the low-frequency fluxes are averaged over a tidal cycle.

At both $\mathrm{B}$ and $\mathrm{C}$, the contribution of $F_{\mathrm{nl}}$ is substantial. The ratio $F_{\mathrm{nl}} / F_{\mathrm{h}}$ is close to 0.6 over the range of fluxes observed (figure 4). The non-hydrostatic flux is smaller but none the less still significant at $\mathrm{B}$, while it is only a small fraction of the total at $\mathrm{C}$, where the high-frequency oscillations begin to interact with the bottom, and so tend to have flatter and wider troughs. Since the non-hydrostatic term peaks over the shoulders of the waves (e.g. Scotti \& Pineda 2004, figure 3), it tends to contribute less once averaged over several waves, even though locally it may still be large.

\subsection{High- and low-frequency fluxes}

A frequent claim is that although the instantaneous values of the depth-averaged energy flux during the passage of NLIWs can be very large, the contribution over time is small, because NLIWs occupy a small fraction of the total cycle. The present analysis does show large instantaneous fluxes. At B it exceeds $9 \mathrm{~kW} \mathrm{~m}^{-1}$, with a median value of $1.2 \mathrm{~kW} \mathrm{~m}^{-1}$ when NLIWs are present. To assess the average flux, we bandpass filtered the time series into high-frequency and low-frequency components (figure 5). The cut-off of the filter was set to separate the NLIW component from the 
rest of the signal. The high-frequency flux is non-zero during about $15 \%$ of the tidal cycle but accounts for about $60 \%$ of the total flux averaged over the entire cycle. While this result cannot be generalized to other areas, it is important to recognize that the energy contained in nonlinear high-frequency internal waves may be an important term in the overall energy baroclinic budget.

\subsection{Dissipation and mixing}

At both sites, energy fluxes are consistently directed towards the west-southwest. Assuming a two-dimensional propagation, we can estimate dissipation levels by subtracting fluxes at each site. To establish a baseline dissipation we consider the lowpassed fluxes first, neglecting the dissipation due to bottom friction, and assuming that the internal dissipation occurs within a region of enhanced shear centred on the pycnocline (about $20 \mathrm{~m}$ thick, based on EOF analysis) extending between B and C. The estimated value is $\epsilon_{k}=10^{-6} \mathrm{~W} \mathrm{~kg}^{-1}$, where we have used a $20 \%$ mixing efficiency to separate $\epsilon_{k}$ from $\epsilon_{p}$. Dissipation associated with the high-frequency component was calculated by integrating the fluxes over 2 hour periods and assuming that dissipation occurs only within the volume of the pycnocline occupied by the packet $(20 \mathrm{~m} \times 3.6 \mathrm{~km})$ during its transit time from B to C (approximatively 5.5 hours). Using a standard parameterization for bottom friction, we estimate $\epsilon_{k}=2.6 \times 10^{-6} \mathrm{~W} \mathrm{~kg}^{-1}$, which is close to values measured with microstructure profilers under similar conditions (Klymak \& Moum 2003). Thus, NLIWs increase the dissipation by only a factor of 2.6. This modest increase suggests that in Massachusetts Bay, waves do not beget large-scale instabilities between B and C. Rather, it is likely that the strain field associated with the waves increases locally the background turbulence.

\section{Conclusion}

We have developed a formalism to calculate baroclinic energy fluxes that applies to both linear and nonlinear internal waves. The centrepiece is an equation that, for the appropriate choice of the reference state, describes conservation of kinetic energy and APE. We have shown that equipartition between kinetic and potential energy does not hold for NLIWs that begin to interact with a shoaling bottom. We also have shown that $\int u^{\prime} p^{\prime} \mathrm{d} z$ underestimates the total energy fluxes by as much as $50 \%$ when NLIWs are present. The linear scaling between the different terms in the total flux and the linear energy flux can be used to estimate the total flux from knowledge of $\int u^{\prime} p^{\prime} \mathrm{d} z$ alone, but more observations are needed to ascertain how robust the correlation is.

We would like to thank R. Camassa, J. H. Fernando, K. Lamb and J. Moum for discussions on energy and mixing in stratified flows. MBIWE98 was supported by the US Geological Survey and the Office of Naval Research. A.S. received support from the Office of Naval Research (N00014-05-1-0361), R.B. from the Walter A. and Hope Noyes Smith Chair on Coastal Oceanography and B.B. from the US Geological Survey.

\section{REFERENCES}

Apel, J. R., BADIEY, M., ChIU, C.-S. et al. 1997 An overview of the 1995 SWARM shallow-water internal wave acoustic scattering experiment. IEEE J. Ocean. Engng 22, 465-500.

Apel, J. R., Ostrovsky, L. A. \& Stepanyants, Y. A. 1995 Internal solitons in the ocean. Tech. Rep. MERCJRA0695. Applied Physics Laboratory, JHU, Baltimore, MD. 
Butman, B., Alexander, P. S., Scotti, A., Beardsley, R. C. \& Anderson, S. 2006 Large internal waves in Massachusetts Bay transport sediment offshore. Cont. Shelf. Res. (in press).

Chang, M. H., Lien, R. C., TAng, T. Y., D’Asaro, E. A. \& Yang, Y. J. 2006 Energy flux of nonlinear internal waves in northern South China Sea. Geophys. Res. Lett. 33, L03607.

Chereskin, T. K. 1983 Generation of internal waves in Massachusets Bay. J. Geophys. Res. 88, 2649-2661.

DudA, T. D., LYNC, J. F., IRISH, J. D. et al. 2004 Internal tide and nonlinear internal wave behavior at the continental slope in the Northern South China Sea. IEEE J. Ocean. Tech. 29, 1105-1130.

Fernando, H. J. S. 1991 Turbulent mixing in stratified fluids. Annu. Rev. Fluid Mech. 23, 455-493.

Helfrich, K. R. \& Melville, W. K. 2006 Long nonlinear internal waves. Annu. Rev. Fluid Mech. 38, 395-425.

Hosegood, P. \& van Haren, H. 2004 Near-bed solibores over the continental slope in the FaeroeShetland Channel. Deep-Sea Res. II 51, 2943-2971.

JACKsON, C. R. 2004 An Atlas of Internal Solitary-like Internal Waves. Global Ocean Associates.

Jeans, D. R. G. \& Sherwin, T. J. 2001 The evolution and energetics of large amplitude nonlinear internal waves on the Portuguese shelf. J. Mar. Res. 59, 327-353.

KLymak, J. M. \& Moum, J. N. 2003 Internal solitary waves of elevation advancing on a shoaling shelf. Geophys. Res. Lett. 30, 2045, doi:10.1029/2003GL017706.

Kunze, R., Rosenfeld, L. K., Carter, G. S. \& GregG, M. C. 2002 Internal waves in Monterey Submarine Canyon. J. Phys. Oceanogr. 32, 1890-1913.

Laurent, L. St. \& Garrett, C. 2002 The role of internal tides in mixing the deep ocean. J. Phys. Oceanogr. 32, 2882-2899.

Lien, R. C., Tang, T. Y., Chang, M. H. \& D’Asaro, E. A. 2005 Energy of nonlinear internal waves in the South China Sea. Geophys. Res. Lett. 32, L03615.

LoRENZ, E. N. 1955 Available potential energy and the maintenance of the general circulation. Tellus 7, 157-167.

Margules, M. 1903 Über die Energie der Stürme. Jahrb. k. k. Zent.-Anst. Meteorol. Erdmagnet. 48, $1-26$.

Moum, J. N. \& Sмyтh, W. D. 2006 The pressure disturbance of a nonlinear internal wave. J. Fluid Mech. 558, 153-177.

Nash, J. D., Alford, M. H. \& Kunze, E. 2005 Estimating internal wave energy fluxes in the ocean. J. Atmos. Ocean. Tech. 22, 1551-1570.

Osborne, A. R. \& Burch, T. I. 1980 Internal solitons in the Andaman Sea. Science 208, 451.

PInEDA, J. 1991 Predictable upwelling and the shore-ward transport of planktonic larvae by internal tidal bores. Science 253, 548.

RAmp, S. R., TANG, T. Y., DudA, T. F. et al. 2004 Internal solitons in the northeastern South China Sea Part I: Sources and deep water propagation. IEEE J. Ocean. Engng 29, 1157-1181.

Scotti, A., Butman, B., Beardsley, R. C., Alexander, P. S. \& Anderson, S. 2005 A lagged beam-to-earth transformation to measure short-wavelength internal waves with an Acoustic Doppler Current Profiler (ADCP). J. Atmos. Ocean. Tech. 22, 583-591.

Scotti, A. \& Pineda, J. 2004 Obervation of very large and steep waves of elevation near the Massachusetts coast. Geophys. Res. Lett. 31, L22307.

Shepherd, T. G. 1993 A unified theory of available potential-energy. Atmos.-Ocean 31, 1-26.

Trask, R. P. \& Briscoe, M. G. 1983 Detection of Massachusetts Bay internal waves by the synthetic aperture radar (SAR) on SEASAT. J. Geophys. Res. 88, 1789-1799.

Winters, K. B. \& D’Asaro, E. A. 1996 Diascalar flux and the rate of fluid mixing. J. Fluid Mech. 317, 179-193.

Winters, K. B., Lombard, P. N., Riley, J. J. \& D’Asaro, E. A. 1995 Available potential energy and mixing in density stratified fluids. J. Fluid Mech. 289, 115-128. 